\title{
Apresentação de vídeo educativo do projeto de extensão: "diagnóstico, tratamento e epidemiologia das doenças da cavidade bucal - LEBU", do Departamento de Odontologia da Universidade Estadual de Maringá - PR
}

Wilton Mitsunari Takeshita*, Lilian Cristina Vessoni Iwaki**, Liogi Iwaki Filho***, Mariliani Chicarelli da Silva**, Neli Pieralisi****, Vanessa Cristina Veltrini*****, Wesley Fernando Ferrari*******

\author{
* Doutor em Radiologia Odontológica - FOSJC/UNESP/São José \\ dos Campos. Professor Adjunto de Estomatologia e Radiologia \\ do Departamento de Odontologia da Universidade Estadual de \\ Maringá - UEM e Faculdade Ingá. Maringá. Paraná. Brasil \\ ** Doutora em Radiologia Odontológica - FOP/UNICAMP/ \\ Piracicaba. Professora Adjunto de Estomatologia e Radiologia \\ do Departamento de Odontologia da Universidade Estadual de \\ Maringá - UEM. Maringá. Paraná. Brasil \\ *** Doutor em Diagnóstico Bucal, professor associado de Cirurgia \\ do Departamento de Odontologia da Universidade Estadual de \\ Maringá \\ **** Mestre em Periodontia USP-Bauru, Professora Assistente de \\ Estomatologia e Radiologia do Departamento de Odontologia da \\ Universidade Estadual de Maringá - UEM. Maringá. Paraná. Brasil \\ ***** Doutora em Patologia pela FOUSP. Professora de Patologia do \\ Departamento de Odontologia da UEM \\ ******* Cirurgião-dentista, graduado pela Universidade Estadual de \\ Maringá
}

\section{RESUMO}

Objetivo: divulgar o Projeto e seus serviços aos municípios da $15^{\mathrm{a}}$ Regional de Saúde, alertar o telespectador a respeito do auto-exame, mostrar ao profissional da Odontologia a importância da iniciativa em se realizar biópsias e exames histopatológicos quando necessário (diante de determinados diagnósticos presuntivos), salientar o valor da interdisciplinaridade na Odontologia, reiterar a importância e grandiosidade dos resultados obtidos quando diferentes esferas (Universidade e Regional de Saúde) trabalham em busca de um bem comum: a qualidade de vida do paciente. Metodologia: para a confecção do vídeo, fo- ram gravadas imagens de palestras, do acolhimento dos pacientes na clínica odontológica, das consultas, dos exames pré-operatórios e dos procedimentos cirúrgicos, bem como dos trabalhos realizados pelos profissionais da assistência social, psicologia, fonoaudiologia, pedagogia e nutrição. Imagens com o trabalho junto à comunidade externa e com a explicação audiovisual do auto-exame foram editadas. Resultados: o trabalho resultou em um vídeo que explorou de forma educativa e dinâmica um trabalho que vem sendo realizado há 15 anos pelo Projeto LEBU, em parceria com a $15^{\text {a }}$ Regional de Saúde. Didaticamente conseguiu explicar a importância em se fazer o 
Apresentação de vídeo educativo do projeto de extensão: "diagnóstico, tratamento e epidemiologia das doenças da cavidade bucal - LEBU”, do Departamento de Odontologia da Universidade Estadual de Maringá - PR • Takeshita WM, Iwaki LCV, Iwaki Filho L, Silva MC, Pieralisi N, Veltrini VC, Ferrari WF

auto-exame bucal em busca de estruturas que não estejam em seu padrão normal. Conclusão: colaborou fortemente para a contemplação de um dos compromissos do LEBU, que é entrelaçar de forma prática e real a Universidade (Departamento de Odontologia) com as necessidades da sociedade $\left(15^{\text {a }}\right.$ Regional de Saúde do Paraná) de forma sensível a seus problemas, colaborando na reflexão, construção e difusão dos valores da cidadania.

\section{DESCRITORES}

Auto-exame. Diagnóstico Precoce. Câncer Bucal.

$\mathbf{H}$ á muitos anos, o ensino na área da Saúde apresenta uma série de lacunas a serem superadas frente à desigualdade social da população, não somente brasileira como mundial, no qual muitos indivíduos não usufruem dos avanços tecnológicos. Sendo assim, a reforma educacional é fundamental para que ocorra uma aprendizagem transformadora dos envolvidos no cenário do sistema básico de saúde. Para que isso ocorra há a necessidade de mudança no perfil dos egressos que passam pelas instituições de ensino.

O curso de Odontologia da Universidade Estadual de Maringá, foi um dos pioneiros a inovar o seu ensino, e nos seus projetos de extensão não poderia ser diferente, onde desde 1995 foi criado um Projeto de extensão inter e multidisciplinar intitulado: "Diagnóstico, tratamento e epidemiologia das doenças da cavidade bucal - LEBU", que atende pessoas portadoras de alguma lesão em boca, desde seu diagnóstico ao tratamento e acompanhamento. Esses pacientes o procuram espontaneamente ou são encaminhados por profissionais da saúde das Unidades Básicas de Saúde (UBS) municipais, o que o tornou referência dentro da $15^{\text {a }}$ Regional de Saúde do Paraná, composta atualmente por 30 municípios.

O Projeto está organizado em ações divididas em três eixos: o primeiro é o eixo assistencial voltado para o diagnóstico e tratamento das lesões que acometem a cavidade bucal, possibilitando o levantamento dos dados epidemiológicos destas doenças. Favorece também ação de apoio psicossocial aos usuários, nos casos necessários, como de cirurgia, comunicação de resultados de exames histopatológicos e encaminhamentos sociais (benefício da prestação continuada, auxílio alimentação); o segundo é o eixo da prevenção que enfoca as atividades de educação e promoção comunitária, compreendendo atividades de divulgação, orientação e prevenção para o bem estar bucal; por último vem o eixo da sistematização do conhecimento, no qual, os acadêmicos dos $3^{\circ}, 4^{\circ}$, e $5^{\circ}$ anos do curso de graduação, residentes em Cirurgia e Traumatologia Bucomaxilofaciais, docentes e técnicos administrativos do Departamento de Odontologia da Universidade Estadual de Maringá e afins, organizam os estudos nos diversos âmbitos que esta temática possibilita para publicações em revistas e/ou congressos, semanas acadêmicas, encontros, seminários e outros meios de divulgação. Existe no Projeto, uma busca constante pela construção permanente da indissociabilidade entre extensão, ensino e pesquisa. ${ }^{13}$

No Projeto são diagnosticados diversas lesões, que segundo a OMS (2002) podem ser subdivididas em cistos odontogênicos e não odontogênicos, tumores odontogênicos, patologias epiteliais, patologias das glândulas salivares, tumores/lesões dos tecidos moles, patologias ósseas, estomatodermopatologias, doenças infecciosas, doenças inflamatórias e outras. ${ }^{15}$ Dentre as diversas lesões podemos citar o câncer bucal devido o seu caráter de alta morbidade.

O número de casos de câncer aumentou consideravelmente em todo o mundo, principalmente a partir do século passado, configurando-se, na atualidade, como um dos mais importantes problemas de saúde pública mundial. ${ }^{20} \mathrm{O}$ câncer bucal possui uma predominância nos países em desenvolvimento, em especial na classe com níveis socioeconômicos mais baixos, ou seja, em pacientes que possuem maiores dificuldades de acesso ao sistema privado de saúde, portanto dependentes do sistema público, no qual costuma ocorrer uma espera longa pelo atendimento, favorecendo um diagnóstico tardio, cujo tratamento é mais agressivo, com um prognóstico desfavorável, reduzindo assim sua qualidade de vida e aumentando as taxas de mortalidade. A estimativa nacional para 2010/2011 o aponta como o $7^{\circ}$ mais incidente, mostrando uma expectativa de 10330 casos novos em homens e 3790 em mulheres. ${ }^{12}$

O quadro atual de risco do câncer bucal no Brasil e suas tendências mostram relevância no âmbito da saúde pública, e evidenciam a necessidade contínua de realizações de pesquisas sobre este tema, buscando informações de qualidade sobre sua distribuição de incidência e mortalidade, as quais são essenciais para o desenvolvimento de políticas de saúde adequadas que visem o seu controle no país. ${ }^{12}$

Além disso, o conhecimento adquirido nas últimas décadas a respeito dos fatores de risco associados à etiopatogenia do câncer vem tornando possível iden- 
tificar quais os indivíduos mais susceptíveis a desenvolver a doença, tornando-os alvo de ações preventivas.

Stahl et al. ${ }^{19}$ (2004), relataram em seu trabalho de pesquisa que campanhas sobre câncer bucal têm contribuído para conscientização da população a respeito dos fatores de risco e colaboram para o aprimoramento do conhecimento dos profissionais de saúde, principalmente os profissionais da Odontologia, com relação a esta patologia. Elango et al. ${ }^{8}$ (2011), acrescentam ainda que condutas de auto-exame de boca têm contribuído para diminuir a prevalência de câncer bucal.

Hertrampf et al. ${ }^{11}$ (2011), relataram em um estudo longitudinal, que a melhora do conhecimento dos Cirurgiões-dentistas a respeito dos fatores que predispõe o câncer bucal contribui para diminuir os valores de prevalência do mesmo. Por isso, o desenvolvimento de um vídeo de apresentação do Projeto LEBU se fez necessário, principalmente no que concerne as ações preventivas de promoção de saúde.

Em vista disso, com finalidade de aprimorar a sistematização do conhecimento e a prevenção, contemplando o segundo e terceiro eixos, foi elaborado uma apresentação em vídeo com os seguintes objetivos: divulgar o Projeto e seus serviços aos municípios da $15^{\text {a }}$ Regional de Saúde, alertar o telespectador a respeito do auto-exame bucal, mostrar ao profissional da Odontologia a importância da iniciativa em se realizar biópsias e exames histopatológicos quando necessário (diante de determinados diagnósticos presuntivos), salientar o valor da inter e multidisciplinaridade na Odontologia, reiterar a importância e grandiosidade dos resultados obtidos quando diferentes esferas (Universidade e Regional de Saúde, por exemplo) trabalham em busca de um bem comum: a qualidade de vida do paciente.

\section{METODOLOGIA}

Após discussões com a equipe inter e multidisciplinar que atua no projeto, para a confecção do vídeo, foram gravadas imagens de palestras, do acolhimento dos pacientes na clínica odontológica, das consultas, dos exames pré-operatórios e dos procedimentos cirúrgicos, bem como dos trabalhos realizados pelos profissionais da assistência social, psicologia, fonoaudiologia, pedagogia e nutrição, já que o projeto é multidisciplinar, levando o aluno a aprender a trabalhar em conjunto com outras áreas da saúde.

A equipe de filmagens também teve acesso a depoimentos de pessoas vinculadas ao Projeto de diferentes formas, como o da coordenadora, o de uma aluna participante durante a graduação e pós-graduação e o depoimento de um paciente. As imagens com o trabalho junto à comunidade externa e com a explicação audiovisual do auto-exame bucal foram inseridas. A rotina das disciplinas de Radiologia, Estomatologia,CirurgiaePatologiatambémforamapresentadas, uma vez que o projeto atua não somente de forma multidisciplinar, mas também interdisciplinar.

Todos os docentes e acadêmicos que apareceram no vídeo concordaram em ter sua imagem vinculada ao Projeto, da mesma maneira que os pacientes exibidos não só concordaram como estimularam a iniciativa, tendo em vista que um maior número de indivíduos terá conhecimento dos cuidados bucais a serem tomados no dia-a-dia e dos serviços oferecidos pelo Projeto.

\section{RESULTADO}

Todo e esforço empregado durante aproximadamente 6 meses, resultou em um vídeo que explorou de forma educativa e dinâmica um trabalho que vem sendo realizado há 15 anos pelo Projeto LEBU, em parceria com a $15^{\text {a }}$ Regional de Saúde. Didaticamente conseguiu explicar a importância para o indivíduo em realizar o auto-exame bucal em busca de estruturas que não estejam em seu padrão normal. Mostrar o esforço da equipe em manter e/ou devolver ao paciente a função normal do sistema estomatognático, por meio da prevenção e promoção da saúde bucal. $\mathrm{O}$ trabalho foi avaliado de maneira positiva. $\mathrm{O}$ plano de filmagens, montado a partir de um texto pré-determinado, facilitou as atividades. A receptividade por parte dos pacientes foi grande e enriquecedora. $\mathrm{O}$ apoio por parte dos acadêmicos e docentes foi crucial para o resultado final. Por fim, a parceria com o Programa Nacional de Reorientação da Formação Profissional em Saúde-Pró-Saúde que disponibilizou recursos para viabilizar a realização do Projeto, possibilitando alcançar os objetivos pretendidos com o vídeo.

\section{DIscussão}

Embora ao longo do tempo, a fim de melhorar a qualidade e abrangência, o modelo de Saúde Pública no Brasil tenha sofrido uma série de mudanças, ainda são necessárias profundas transformações, e para que isso venha a ocorrer, é preciso haver também transformações no processo de formação e desenvolvimento dos profissionais da área da saúde, que somente ocorrerá se mudarmos a forma de como cuidar, tratar e acompanhar a saúde de nossos pacientes, conseguido por meio de mudanças também nos modos de 
Apresentação de vídeo educativo do projeto de extensão: "diagnóstico, tratamento e epidemiologia das doenças da cavidade bucal - LEBU”, do Departamento de Odontologia da Universidade Estadual de Maringá - PR • Takeshita WM, Iwaki LCV, Iwaki Filho L, Silva MC, Pieralisi N, Veltrini VC, Ferrari WF

ensinar e aprender. Assim, é fundamental que os atores envolvidos no ensino, principalmente na área da saúde, tenham em mente que o seu papel é formar cidadãos que possam enxergar o paciente como um todo, e se preocupe não somente em tratar as doenças, mas principalmente em preveni-las, devendo os envolvidos com a academia, se aproximarem cada vez mais da comunidade e do serviço.

Comojá mencionado, não somente em função da busca de aproximação com a comunidade, o projeto de extensão: "Diagnóstico, tratamento e epidemiologia das doenças da cavidade bucal - LEBU”, desde 1995, executa diagnósticos, tratamento e/ou acompanhamento de pessoas portadoras de alguma lesão em boca, estando dividido em três eixos:

- assistencial,

- prevenção e

- sistematização do conhecimento.

O eixo assistencial do Projeto contempla o diagnóstico e tratamento do indivíduo com lesões bucais e necessita de uma equipe multidisciplinar que trabalha de forma integrada, objetivando a eliminação da doença com manutenção da qualidade de vida do paciente. Fazem parte dessa equipe de profissionais: cirurgiões-dentistas, médicos (cirurgiões de cabeça e pescoço, oncologistas, radioterapeutas), enfermeiros, psicólogos, fonoaudiólogos, nutricionistas, assistentes sociais, sem destacar nenhuma dessas figuras, que em suas áreas de competência, trabalham e cooperam no atendimento ao paciente. ${ }^{2,5}$ Neste eixo, os acadêmicos aprendem a trabalhar em equipe e visualizar o paciente como um todo e não apenas uma boca a ser tratada.

Com relação ao segundo eixo do Projeto, o da prevenção, os acadêmicos desenvolvem uma visão moderna da área que é a valorização da saúde e não da doença, realizando isso por meio de ações educativas, tentando mudar valores e comportamentos dos pacientes. Os participantes do Projeto têm o dever de orientar os pacientes quanto ao malefício do fumo, álcool, exposição excessiva ao sol, da importância de uma boa higiene e saúde bucal, da integridade dos dentes e aparelhos protéticos, os benefícios da alimentação balanceada, orientações quanto à importância e técnica de auto-exame bucal a fim de se evitar o aparecimento de inúmeras doenças. ${ }^{17}$

Com relação à prevenção para a melhoria da expectativa de vida da população, medidas devem ser tomadas a fim de incentivar atitudes preventivas em relação ao câncer, como o controle do consumo de tabaco e álcool, a adoção de dieta rica em vegetais e frutas frescas, além de uma maior qualidade nos serviços de diagnóstico e tratamento dos pacientes com câncer bucal e de faringe. ${ }^{1,3,4,21} \mathrm{O}$ exame clínico da boca, principalmente dos pacientes com hábitos nocivos como álcool e cigarro, deve ser sistemático e indivíduos com lesões suspeitas devem ser imediatamente encaminhados à consulta especializada em centros de referência para realização dos procedimentos diagnósticos necessários. É importante também que o fumo e o álcool, que são fatores passíveis de intervenção por programas de prevenção primária que realizem ações legislativas, educativas e econômicas com o objetivo de reduzir o acesso ou a exposição da população a esses fatores. ${ }^{6,14,15,17}$

Segundo Eadie $e t a l .^{7}$ (2009), três são os fatores que desestimulam o paciente a procurar auxílio e orientação profissional:

- seu pequeno conhecimento a respeito do assunto,

- sua pouca percepção do risco e

- seu medo a respeito dos aspectos negativos desse diagnóstico.

Por conseguinte, os envolvidos no Projeto, mais especificamente os acadêmicos, aprendem que todos os membros de uma equipe multiprofissional integrada atuam não só curativamente, mas podem ressaltar os aspectos preventivos das lesões bucais; bem como, a busca de uma boca saudável pode ser favorável para o desempenho do tratamento, visto que é íntima a relação de condições inflamatórias bucais crônicas e a saúde geral.

Ainda no segundo eixo tem-se a promoção de saúde, no qual Stahl et al..$^{19}$ (2004), realizaram uma pesquisa para avaliar resultados de campanhas de prevenção do câncer bucal na população e nos profissionais da Odontologia. Os autores concluíram que a campanha contribuiu para a população atentar para alterações de boca e quando detectada entrar em contato com profissionais da área, haja vista que desconheciam o fato de que os Cirurgiões-dentistas são responsáveis pelo exame de câncer bucal. Além disso, a população se apresentou mais conhecedora da importância do diagnóstico precoce para um melhor prognóstico da doença. Com relação aos profissionais da Odontologia a campanha contribuiu para mudanças de rotina clínica, principalmente na discussão da doença com seus pacientes no que concerne o diagnóstico precoce e uma atenção maior para 
Apresentação de vídeo educativo do projeto de extensão: "diagnóstico, tratamento e epidemiologia das doenças da cavidade bucal - LEBU”, do Departamento de Odontologia da Universidade Estadual de Maringá - PR • Takeshita WM, Iwaki LCV, Iwaki Filho L, Silva MC, Pieralisi N, Veltrini VC, Ferrari WF

pequenas lesões de boca. Nesta linha de conclusão, o material em vídeo elaborado contempla algumas vertentes desse processo, apresentando o trabalho desenvolvido no Projeto pelos diferentes profissionais participantes e ensinado o paciente a valorizar e realizar o auto-exame bucal.

Na sistematização do conhecimento contemplando o terceiro eixo, trabalhos de levantamento epidemiológicos são necessários, uma vez que diferentes grupos populacionais podem apresentar diferentes prevalências para determinadas lesões. ${ }^{15}$ No trabalho de Rioboo-Crespo et al. ${ }^{18}$ (2005), estudando a prevalência de lesões bucais em crianças, concluíram que a candidíase bucal apresentou maior prevalência, sendo esta justificada em função da presença de um sistema imunológico imaturo nas crianças.

Ainda na sistematização do conhecimento, Gajendra et $a l^{10}$ (2006) relataram em seu trabalho de pesquisa que na formação dos profissionais da Odontologia existem lacunas no conhecimento de fatores de risco do câncer bucal. Cannick et al. ${ }^{4}$ (2005), avaliaram o grau de conhecimento dos alunos de uma Faculdade de Odontologia do Sul da Califórnia a respeito do câncer bucal, e observaram que apesar do aumento do nível de conhecimento dos alunos com o avanço das séries, o ensino deve dar mais ênfase também na educação a respeito do tema câncer bucal, pois os índices de mortalidade tendem a diminuir se os Cirurgiões-dentistas tiverem maior conhecimento dos fatores de prevenção e detecção precoce do câncer bucal. Além disso, no trabalho de Epstein et al. ${ }^{9}$ (2008) os autores relataram a importância de um trabalho realizado com estudantes de Odontologia, em que realizaram uma campanha de uma semana para avaliação de pacientes e esclarecimentos a respeito do câncer bucal. Eles concluíram que a semana foi importante para reforçar a análise da região de cabeça e pescoço e tecidos moles bucais por parte dos alunos e divulgação para a comunidade a respeito do diagnóstico precoce e auto-exame. Sendo este tipo de campanha rotina para os nossos acadêmicos.

Frente a tudo o que foi exposto, para maiores esclarecimentos, contemplando o segundo e terceiro eixos, buscou-se o desenvolvimento de um vídeo com finalidade de apresentar aos profissionais da saúde e à população, o Projeto LEBU e suas competências, sendo uma das várias ferramentas utilizadas como mecanismo de divulgação e busca por uma mudança de conduta frente a alterações bucais. $O$ vídeo consta de depoimentos de pessoas vinculadas ao Projeto, como o da coordenadora, o de uma aluna participante durante a graduação e pós-graduação e o depoimento de um paciente. Apresenta também imagens com trabalho junto à comunidade externa e com a explicação audiovisual do auto-exame. A rotina das disciplinas de Radiologia, Estomatologia, Cirurgia e Patologia.

A produção do material envolveu seis meses de trabalho entre gravações internas (imagens clínicas), externas e a edição do vídeo, no qual foi possível perceber a importância da dedicação, atenção e cuidado por parte da equipe de filmagens e por parte dos participantes do Projeto, já que independente de imagens e pessoas, o protagonista deveria sempre ser o Projeto LEBU. Isto se refletirá diretamente no telespectador, motivo principal do trabalho.

\section{CONClusóES}

Assim, fica nítida a importância da parceria entre

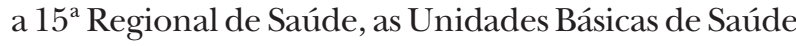
dos municípios participantes, o Projeto LEBU, a Clínica Odontológica da Universidade Estadual de Maringá, os acadêmicos, os docentes, os demais profissionais envolvidos, os pacientes e acima de tudo a viabilização de recursos financeiros feito pelo Programa Nacional de Reorientação da Formação Profissional em Saúde - Pró-Saúde, sem o qual todo o trabalho acima descrito não seria possível. $\mathrm{O}$ material desenvolvido salienta a importância do auto-exame bucal e também divulga o Projeto, cuja busca pela preocupação com o ser humano como um todo. A ausência de quaisquer partes acima citadas na engrenagem formada para a realização da tarefa resultaria no desvio do objetivo ou até mesmo na impossibilidade de conclusão da mesma. Foi um trabalho que possibilita pontos positivos principalmente ao telespectador, alvo principal do trabalho. Com isso colaborando fortemente para a contemplação de um dos compromissos do LEBU, que é entrelaçar de forma prática e real a Universidade (Departamento de Odontologia) com as necessidades da sociedade ( $15^{\text {a }}$ Regional de Saúde do Paraná) de forma sensível a seus problemas, colaborando na reflexão, construção e difusão dos valores da cidadania.

\section{AGRADECIMENTOS}

À Universidade Estadual de Maringá instituição em que foi desenvolvido o vídeo, à Imagem Vídeo produtora do vídeo, ao PRÓ-SAÚDE, aos docentes, funcionários, alunos e pacientes que tornaram esse Projeto viável. 
Apresentação de vídeo educativo do projeto de extensão: "diagnóstico, tratamento e epidemiologia das doenças da cavidade bucal - LEBU”, do Departamento de Odontologia da Universidade Estadual de Maringá - PR • Takeshita WM, Iwaki LCV, Iwaki Filho L, Silva MC, Pieralisi N, Veltrini VC, Ferrari WF

\section{ABSTRACT}

Presentation of an educational video of the extension project, “diagnosis, epidemiology and treatment of oral cavity diseases - LEBU," Department of Dentistry, State University of Maringá - PR

Objective: To present the project and its services to cities in the $15^{\text {th }}$ Regional Health Area, alert viewers to self-examinations, show the dental professional the importance of the initiative to perform biopsies and histopathological exams when needed (presumptive diagnosis), emphasize the value of interdisciplinarity in dentistry, reiterate the importance and impressiveness of the results obtained when different spheres (University and Regional Health area) work in pursuit of a common good: the quality of life of patients. Methodology: In order to make the video, images were recorded of lectures, the reception of patients in the dental clinic, consultations, preoperative examinations and surgical procedures, and the work done by professionals in social work, psychology, speech therapy, teaching and nutrition. Images with the outside community and the visual explanation of self-examination were also included. Results: The work resulted in a video that instructionally and dynamically explored the work being done by the LEBU project for the past 15 years, in partnership with the $15^{\text {th }}$ Regional Health area. The video was able to render a didactic explanation of the importance of performing selfexamination in search of structures that do not follow a normal pattern. Conclusion: The video greatly contributed to highlighting one of the LEBU commitments, which is to dovetail, in a practical and real manner, the University (Department of Dentistry) with the needs of society $\left(15^{\text {th }}\right.$ Regional Health area of Paraná), demonstrating sensitivity to the population's health problems by collaboration toward the reflection, construction and dissemination of citizenship values.

\section{DESCRIPTORS}

Self-Examination. Early Diagnosis. Mouth Neoplasms.

\section{REFERÊNCIAS BIBLIOGRÁFICAS}

1. Adair T, Hoy D, Dettrick Z, Lopez AD. Trends in oral, pharyngeal and esophageal cancer mortality in Australia: the comparative importance of tobacco, alcohol and other risk factors. Aust N Z J Public Health. 2011;35(3):212-9.

2. Almeida FCS, Vaccarezza GF, Cazal C, Benedethe APF, Pinto
Junior DS, Tavares MR et al. Avaliação odontológica de pacientes com câncer de boca. Pré e pós tratamento oncológico - uma proposta de protocolo. Pesqui bras odontopediatria clín integr. 2004;4(1):25-31.

3. Bunnell A, Pettit N, Reddout N, Sharma K, O'Malley S, Chino M, Kingsley K. Analysis of primary risk factors for oral cancer from select US states with increasing rates. Tob Induc Dis. 2010;8(1):5.

4. Cannick GF, Horowitz AM, Drury TF, Reed SG, Day TA. Assessing oral cancer knowledge among dental students in South Carolina. Am Dent Assoc. 2005;136(3):373-8.

5. Costa EG, Miglioratti CA. Câncer bucal: avaliação do tempo decorrente entre a detecção da lesão e o início do tratamento. Rev Bras de Cancerologia. 2001 Jul-Ago-Set;47 (3):283-9.

6. Cruz GD, Ostroff JF, Kumar JV, Gajendra S. Preventing and detecting oral cancer Oral health care providers' readiness to provide health behavior counseling and oral cancer examinations J Am Dent Assoc. 2005;136(5):594-601.

7. Eadie D, MacKintosh AM, MacAskill S, Brown A. Development and evaluation of an early detection intervention for mouth cancer using a mass media approach. British Journal of Cancer. 2009;101:73-9.

8. Elango KJ, Anandkrishnan N, Suresh A, Iyer SK, Ramaiyer SK, Kuriakose MA. Mouth self-examination to improve oral cancer awareness and early detection in a high-risk population. Oral Oncol. 2011 Jun 4. [Epub ahead of print].

9. Epstein JB, Villines D, Drahos G, Kaufman E, Gorsky M. Oral lesions in patients participating in an oral examination screening week at an urban dental school. J Am Dent Assoc. 2008;139(10):1338-44.

10. Gajendra S, Cruz GD, Kumar JV. Oral Cancer Prevention and Early Detection: Knowledge, Practices, and Opinions of Oral Health Care Providers in New York State J Cancer Educ. 2006;21 (3):157-62.

11. Hertrampf K, Wenz HJ, Koller M, Grund S, WiltfangJ. The oral cancer knowledge of dentists in Northern Germany after educational intervention. Eur J Cancer Prev. 2011 May 26. [Epub ahead of print].

12. INCA. Estimativa 2010 - Incidência de câncer no Brasil. Disponível em: http://www.inca.gov.br/estimativa/2010. Acesso em 06 de junho de 2011.

13. Iwaki LCV, Chicarelli M, Takeshita WM, Pieralisi N, Ferreira GZ. Estratégias multidisciplinares de promoção de saúde em portadores de neoplasias bucais malignas desenvolvidas por projetos de extensão da Universidade Estadual de Maringá. Rev Conexão UEPG. 2010; 5 (1):84-9.

14. McCullough MJ, Prasad G, Farah CS. Oral mucosal malignancy and potentially malignant lesions: an update on the epidemiology, risk factors, diagnosis andmanagement. Aust Dent J 2010;55:(1 Suppl): 61-5. 
Apresentação de vídeo educativo do projeto de extensão: "diagnóstico, tratamento e epidemiologia das doenças da cavidade bucal - LEBU", do Departamento de Odontologia da Universidade Estadual de Maringá - PR • Takeshita WM, Iwaki LCV, Iwaki Filho L, Silva MC, Pieralisi N, Veltrini VC, Ferrari WF

15. Mujica V, Rivera H, Carrero M. Prevalence of oral soft tissue lesions in an elderly venezuelan population. Med Oral Patol Oral Cir Bucal. 2008;13(5):270-4.

16. Natarajan E, Eisenberg E. Contemporary concepts in the diagnosis of oral cancer and precancer. Dent Clin North Am. 2011;55(1):63-88.

17. Neville BW, Day TA. Oral Cancer and Precancerous Lesions. CA Cancer J Clin 2002;52:195-215.

18. Rioboo-Crespo MR, Planells-del Pozo P, Rioboo-García R. Epidemiology of the most common oral mucosal diseases in children. Med Oral Patol Oral Cir Bucal 2005;10:376-87.

19. Stahl S, Meskin LH, Brown LJ. The American Dental Associa- tion's oral cancer campaign The impact on consumers and dentists. J Am Dent Assoc. 2004;135(9):1261-7.

20. Torres-Pereira C. Oral cancer public policies: is there any evidence of impact? Braz Oral Res. 2010;24:37-42.

21. Zygogianni AG, KyrgiasG, Karakitsos P, Psyrri A, Kouvaris J, Kelekis N, Kouloulias V. Oral squamous cell cancer: early detection and the role of alcohol and smoking. Head \& Neck Oncology. 2011;3:1-12.

Recebido em 07/07/2011

Aceito em 25/07/2011 